\title{
Probing Depth Study of Conversion Electron/He Ion Yield XAFS Spectroscopy on Strontium Titanate Thin Films
}

\author{
Etsuya Yanase*1, Iwao Watanabe*2, Makoto HaRadA*2, Masao TaKahaShI*3, \\ Yoshinori DAKE*4 and Yasushi HIROSHIMA*4 \\ *1 The New Industry Research Organization, 1-5-2 Minatojima-minamimachi, Chuo, Kobe 650-0047, Japan \\ *2 Department of Chemistry, Graduate School of Science, Osaka University, Toyonaka, Osaka 560-0043, Japan \\ *3 Institute of Scientific and Industrial Research, Osaka University, Ibaraki, Osaka 567-0047, Japan \\ *4 Kanto Technical Institute, Kawasaki Heavy Industries, Ltd, 118 Futatsuzuka, Noda, Chiba 278-8585, Japan
}

\begin{abstract}
The probing depth of conversion electron/He ion yield XAFS methods has been studied in order to apply these methods to estimate the local distortion of strontium titanate thin films on thick substrates. Several strontium titanate thin film samples with different thicknesses were prepared and the edge-jump amplitudes at both the $\mathrm{Sr}$ and $\mathrm{Ti} \mathrm{K}$-edges were obtained. The probing depths were estimated to be $300 \mathrm{~nm}$ for the Sr K-edge and $30 \mathrm{~nm}$ for the Ti K-edge by the conventional exponential fit. The XAFS spectra and the probing depths measured by conversion electron and He ion yield methods is much the same, though the background curves are different.
\end{abstract}

Keywords X-Ray absorption fine structure, conversion electron yield, probing depth, strontium titanate, thin film

High permittivity dielectric thin films, such as $\mathrm{SrTiO}_{3}$, are excellent candidate materials for a capacitor in dynamic random access memory (DRAM). In real applications of such dielectric thin films, any lattice distortion can seriously affect its electric performance. It is known that the dielectric constant of a strontium titanate $\left(\mathrm{SrTiO}_{3}\right)$ thin film deposited onto a thick substrate decreases along with a decrease in the thickness due to lattice distortion caused by internal stress. ${ }^{1}$ It is expected that under internal stress due to lattice distortion the geometrical structure of the thin film may be deformed atomically. It is difficult to detect such a local atomic distortion by an X-ray diffraction technique, while an X-ray absorption fine structure (XAFS) technique might be a better tool. However, since XAFS spectroscopy is basically an absorption spectroscopy, its application to thin films formed on a thick substrate or thick single crystalline material is not easy. For studying such a thin film, a surface sensitive technique is more desirable, and the most convenient method applicable is the total conversion electron/He ion yield (CEY/CIY) XAFS method. In order to promote studies of thin films by this technique, knowledge of the probing depth of the technique is crucial. ${ }^{2-4}$ However, the experimental data available on the probing depths are quite scarce, especially for high energy absorption edges, such as $\mathrm{Sr} \mathrm{K}$-edge or for compounds.

We report here on the probing depths of the CEY/CIY XAFS technique for the $\mathrm{Sr}$ and $\mathrm{Ti}$ K-edges of $\mathrm{SrTiO}_{3}$. The edge-jump currents at both the $\mathrm{Sr}$ and Ti K-edges have been measured as a function of the film thickness of $\mathrm{SrTiO}_{3}$, and the probing depths have been calculated.
The difference between the spectra obtained by the CEY and CIY methods is also reported.

\section{Experimental}

Figure 1 shows a schematic diagram of the CEY/CIY XAFS apparatus. When a sample placed in atmospheric He gas is irradiated with X-ray photons, Auger electrons are ejected from the sample surface in proportion to the photon absorption, and there ionize He atoms.

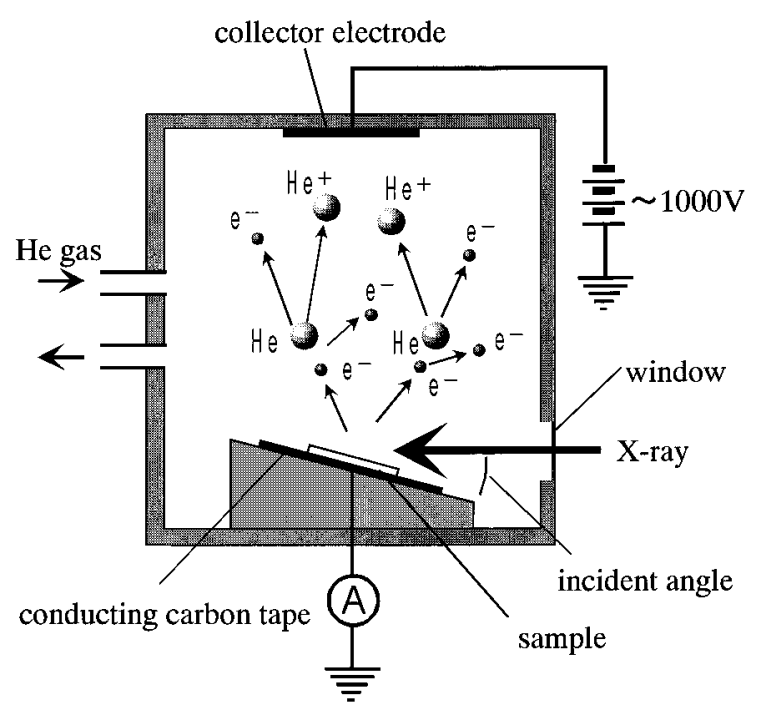

Fig. 1 Schematic diagram of the CEY/CIY XAFS apparatus. 
The Auger electron has a few $\mathrm{keV}$ of kinetic energy, or even more. For example, Sr and Ti KLL Auger electrons have 12 and $3.9 \mathrm{keV}$ energy, respectively. One Auger electron, therefore, produces a bunch of $\mathrm{He}^{+}$ions and secondary electrons; thus, the Auger current is amplified by the presence of $\mathrm{He}$ gas. The electrons or $\mathrm{He}$ ions so generated can be collected by using an electrode. A bias voltage was applied to a collector electrode made of a piece of carbon tape. The sample (10 $\mathrm{mm} \times 10 \mathrm{~mm} \times 1 \mathrm{~mm}$ ) was placed on conductive carbon tape connected to a current amplifier (Keithley 427). It is important to make the carbon tape much larger than the sample size in order to detect the CEY/CIY current from insulator samples. After the CEY/CIY cell was set up on an $x / z / \theta / \phi$ stage, the sample position and angle with respect to the incident X-ray beam could be adjusted from the outside of the experiment hatch. A computer was used to control all axes of stage. The signal intensity as a function of any axis could be graphically displayed on the computer screen in order to assist in finding the best sample setting. The inner cell body was constructed from acrylic plastics; where good electrical insulation was necessary PTFE was used.

Thin films of $\mathrm{SrTiO}_{3}$ were epitaxially grown on $\mathrm{LaAlO}_{3}\left(\begin{array}{lll}1 & 0 & 0\end{array}\right)$ single crystal substrates by a laser deposition method. ${ }^{5}$ Samples with thicknesses from $1 \mathrm{~nm}$ to $100 \mathrm{~nm}$ were prepared. The thickness of the films was confirmed by RHEED oscillation for $1-10 \mathrm{~nm}$ thick samples, by a calculation using the deposition rate for $25-100 \mathrm{~nm}$ samples, and by a contact type displacement gauge for $50-100 \mathrm{~nm}$ samples. The orientation and the crystallinity of the films were determined by an $\mathrm{X}$-ray diffractometer (XRD) and a reflective high-energy electron diffractometer (RHEED). The XRD patterns indicate that the films are preferentially oriented to the $\left(\begin{array}{lll}1 & 0 & 0\end{array}\right)$ plane parallel to the substrate surface, and sharp peak RHEED patterns of the films show that the films are grown epitaxially on the substrate and that the film surface is very smooth.

All measurements were made at beamlines BL-7C ${ }^{6}$ and $\mathrm{BL}-12 \mathrm{C}^{7}$ of the Photon Factory, High Energy Accelerator Research Organization (KEK) in Tsukuba. The X-ray beam was monochromatized by using $\operatorname{Si}(11$ 1) double crystal monochromators, and its second crystal was sagittally bent to focus the beam horizontally (BL-7C), or the beam was focused by using a Rh-coated bent cylindrical mirror (BL-12C). For measurements of the Ti K-edge absorption spectra, the higher harmonics were rejected by using a double quartz mirror system (BL-7C). The beam intensity was monitored with a nitrogen gas ionization chamber of $4 \mathrm{~cm}$ in path length.

\section{Results and Discussion}

Figure 2 compares the X-ray absorption spectrum of $\mathrm{SrTiO}_{3}$ powder at the $\mathrm{Sr}$ K-edge $(16094 \mathrm{eV})$ taken by
CIY at an incidence angle of 5 degree with that taken by the transmission method in order to confirm the validity of our apparatus. The powder sample for CIY was spread on carbon tape. These spectra are exactly the same, except for the background curves. The difference in the background originated from a different energy dependence of the background signal for the two methods. The same spectral data from the two methods indicate that the CIY is a very convenient method for obtaining XAFS spectra of powder samples, since no sample preparation procedures are necessary; the tedious preparation procedures are grinding the sample to a fine powder, mixing with a matrix material to obtain proper sample thickness, and homogeneous packing into a sample holder or pressing to form a plate. It should also be noted that the XAFS spectra of insulator samples, such as a $\mathrm{SrTiO}_{3}$ single crystal plate (10 $\mathrm{mm} \times 10 \mathrm{~mm} \times 1 \mathrm{~mm})$, could be obtained when a wider carbon sheet was used as the sample holder, as shown in Fig. 1. This is because the charged particles, $\mathrm{He}$ ions and secondary electrons, present in the space between the sample and the collector electrode effectively neutralize the surface charge on the sample.

The XAFS spectra of a $100 \mathrm{~nm}$ thick $\mathrm{SrTiO}_{3}$ film were obtained by both CEY and CIY methods at an incidence angle of 5.5 degree, and are compared in Fig. 3. The result indicates that the signal to background ratio (S/B) of the CIY spectrum is larger than that of the CEY because of the contribution of low energy electrons to the CEY current, and that both detection methods produced perfectly the same results.

In order to evaluate the probing depth of the present technique, several $\mathrm{SrTiO}_{3}$ thin film samples with different thicknesses were prepared and the edge-jump amplitudes at both $\mathrm{Sr}$ and Ti K-edges were obtained by both the CEY and CIY methods at an incident angle of 40 degree. The edge-jump amplitudes with both methods were the same. In Fig. 4 are plotted the edge-jump amplitudes $(\delta \mu)$ by the CIY method for (a) the Sr Kedge and (b) the Ti K-edge with respect to the film thickness. The edge-jump amplitude is normalized by the value for a $\mathrm{SrTiO}_{3}$ single crystal sample. The prob-

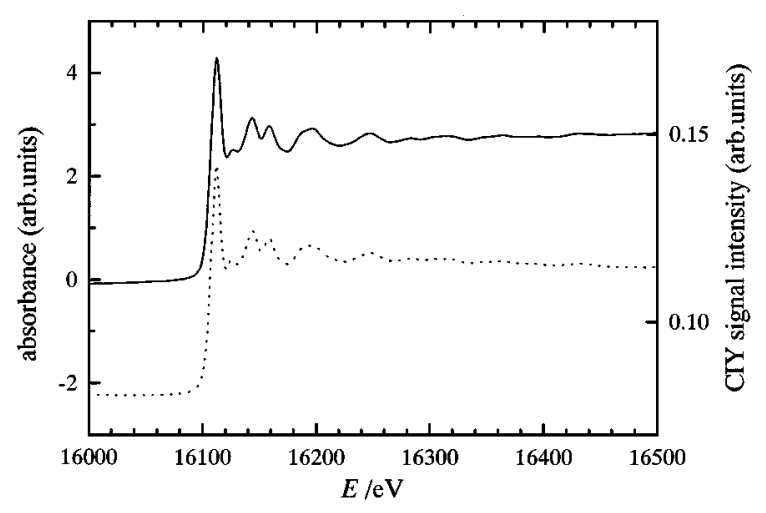

Fig. 2 Sr K-edge XAFS spectra of $\mathrm{SrTiO}_{3}$ powder by CIY (-) and transmission method (---). 


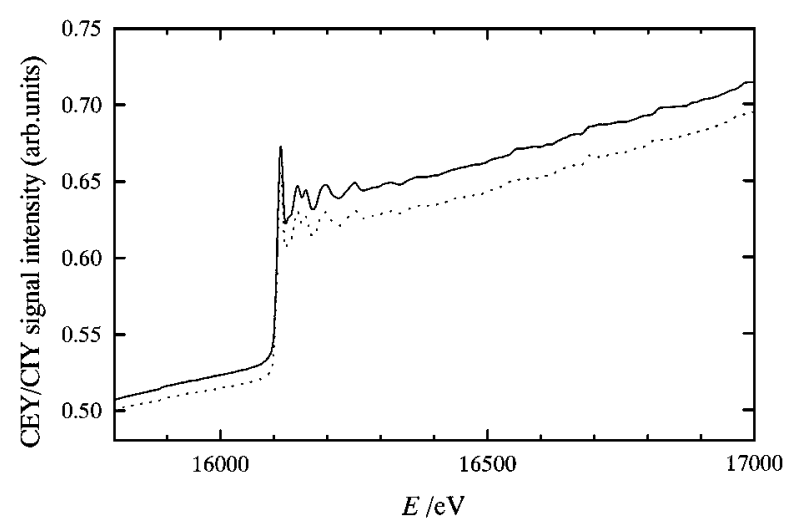

Fig. 3 Sr K-edge XAFS spectra of a $100 \mathrm{~nm} \mathrm{SrTiO}_{3}$ thin film by CEY (-) and CIY (---) method.

ing depths are estimated to be $300 \mathrm{~nm}$ for the $\mathrm{Sr} \mathrm{K}$ edge and $30 \mathrm{~nm}$ for the Ti K-edge by adopting the following equation:

$$
P(d)=\int_{0}^{d} \mathrm{e}^{-x / d} \mathrm{~d} x
$$

where $d$ is a film thickness (in nm) and $P(d)$ the edgejump amplitude for a film of thickness $d$. The best fit to the experimental points is indicated by solid line in Fig. 4. The probing depths are determined by conventional exponential fit here, though experimental evidence has been reported for non-exponential signal attenuation. ${ }^{2}$ The formula for estimating the probing depth has been suggested by Schroeder ${ }^{4}$ as follows:

$$
P(d)=\int_{0}^{d} 0.76 \cdot\left(1-\frac{2 x}{R_{\mathrm{B}}}\right) \cdot \exp \left(-\frac{2.7 x}{R_{\mathrm{B}}}\right) \mathrm{d} x
$$

where $d$ is the film thickness (in $\mathrm{nm}$ ), $R_{\mathrm{B}}$ the Bethe range (in $\mathrm{nm}$ ), and $P(d)$ the edge-jump amplitude for a film with thickness $d$. Shroeder's model is based on
Auger electron depth distribution functions (DDFs) derived from Monte-Carlo trajectory simulations. This model allows quick estimates of probing depth information. We carried out an estimation of $P(d)$ using only Sr or Ti KLL Auger electron, because the charge multiplication in the gas phase weights the signal strongly towards the energetic Auger contribution. Bethe ranges were calculated by summing the modified Bethe-law expression:8,9

$$
\begin{aligned}
R_{\mathrm{B}}=\frac{1}{7850} \int_{E_{0}}^{40 \mathrm{eV}} \frac{E}{\sum_{i=1}^{3} \frac{Z_{i} \rho_{i}}{M_{i}} \ln \left(\frac{J_{i}}{1.166\left(E+t J_{i}\right)}\right)} \mathrm{d} E \\
J_{i}=\left[\begin{array}{ll}
\left.9.76 \cdot Z_{i}+\frac{58.5}{Z_{i}^{0.19}}\right] & \text { for } Z_{i} \geq 13 \\
=11.5 Z_{i} & \text { for } Z_{i} \leq 12
\end{array}\right\}
\end{aligned}
$$

where $E$ is the kinetic energy of the electron (in $\mathrm{eV}$ ), $E_{0}$ the initial electron energy (Sr or Ti KLL Auger electron energy in $\mathrm{eV}$ ), $\rho_{i}$ the density (in $\mathrm{g} / \mathrm{cm}^{3}$ ), $Z_{i}$ the atomic number, $J_{i}$ the mean ionization potential of the material (in $\mathrm{eV}$ ), $R_{B}$ the Bethe range (in $\mathrm{nm}$ ), and $M_{i}$ the atomic weight per a molecule (in $\mathrm{g} / \mathrm{mol}$ ); $i=1,2$, and $3 \mathrm{is} \mathrm{Sr}$, $\mathrm{Ti}$, and $\mathrm{O}$ atom, respectively. The summation of Eq. (2) is carried out over all components ( $\mathrm{Sr}, \mathrm{Ti}$ and $\mathrm{O}$ ). The equations were calculated using the parameters $\rho=5.11$ and $t=0.85$. The values obtained by Schroeder's model are indicated in Fig. 4 by the dotted line, which excellently reproduces the signal intensity of CIY experiments. The difference in the probing depth for the $\mathrm{Sr}$ and Ti K-edges is due to the difference in kinetic energy of the Auger electrons from these atoms.

The CEY/CIY method was used to obtain XAFS spectra for $\mathrm{SrTiO}_{3}$ thin films on a thick substrate on both $\mathrm{Sr}$ and $\mathrm{Ti} \mathrm{K}$-edges. The probing depth of this

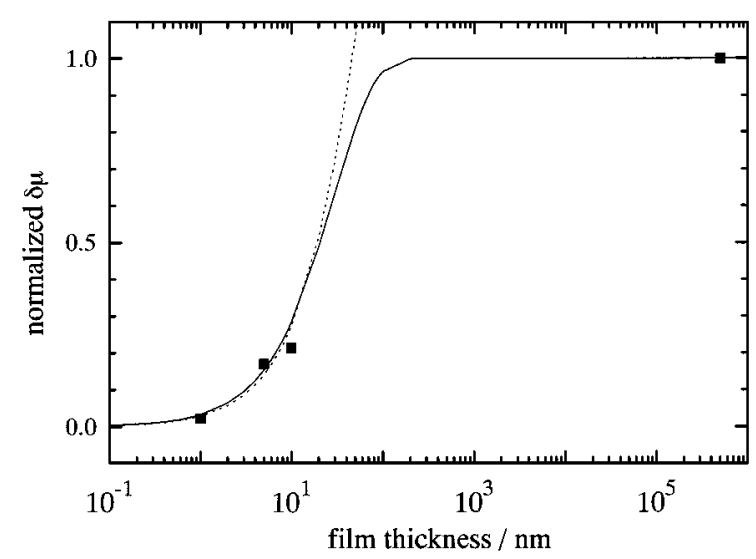

Fig. 4 Edge-jump amplitudes of the XAFS spectra for $\mathrm{SrTiO}_{3}$ thin films plotted against the film thickness; (a) at $\mathrm{Sr}$ K-edge and (b) Ti K-edge energies. The experiments were performed by applying a bias voltage of $-500 \mathrm{~V}$ to the collector electrode and the X-ray incidence angle was $40^{\circ}$. (---) result calculated with the Auger yield model by Schroeder. ${ }^{4}$ 
method was experimentally evaluated by measuring the edge-jumps for several films with different thicknesses. The depth evaluated by fitting an exponential function to the experimental data was $300 \mathrm{~nm}$ for the $\mathrm{Sr} \mathrm{K}$-edge and $30 \mathrm{~nm}$ for the Ti K-edge. The $\mathrm{S} / \mathrm{B}$ ratio from the CIY method was found to be greater than that of the CEY method, though the probing depths and spectral features obtained from both methods were quite the same.

We are indebted to Dr. Hitoshi Tabata, Institute of Science and Technology, Osaka University for preparing the samples and useful discussion. This work was performed under the approval of the Photon Factory Program Advisory Committee (Proposal Nos. 93G160 and 95G223).

\section{References}

1. K. Abe and S. Komatsu, Jpn. J. Appl. Phys., 31, 2985 (1992).

2. A. Erbil, G. S. Cargill III, R. Frahm and R. F. Boehme, Phys. Rev. B, 37, 2450 (1988).

3. S. L. M. Schroeder, G. D. Moggridge, R. M. Ormerod, T. Rayment and R. M. Lambert, Surf. Sci., 324, L371 (1995).

4. S. L. M. Schroeder, Solid State Comm., 98, 405 (1996).

5. T. Tomio, H. Miki, H. Tabata, T. Kawai and S. Kawai, J. Appl. Phys., 76, 5886(1994).

6. M. Nomura, A. Koyama and M. Sakurai, KEK Report 91-1 (1991).

7. M. Nomura and A. Koyama, KEK Report 95-15 (1996).

8. D. C. Joy and S. Luo, Scanning, 11, 176 (1989).

9. R. Herrmann and L. Reimer, Scanning, 620 (1984).

(Received October 19, 1998) (Accepted January 14, 1999) 